\title{
From isotropic turbulence in triply periodic cubic domains to sheared turbulence with inflow/outflow
}

\author{
Chandru Dhandapani $\odot^{*}$ and Guillaume Blanquart $\odot$ \\ California Institute of Technology, Pasadena, California, USA \\ (Received 8 October 2019; accepted 4 November 2020; \\ published 17 December 2020)
}

\begin{abstract}
Homogeneous shear turbulence (HST) is an idealized version of the shear turbulence observed in practical free shear flows, and can be simulated using simple computational domains. One of the numerically efficient configurations to simulate turbulent flows is to use triply periodic domains. However, owing to the mean streamwise velocity being nonhomogeneous, periodic boundary conditions cannot be used along one of the directions. Several studies included shear periodic boundary conditions in the cross-stream direction. However, in these simulations, the turbulence statistics grew exponentially with time, whereas the turbulence observed in free shear flows is statistically stationary. In Dhandapani et al. [Phys. Rev. Fluids 4, 084606 (2019)], the authors fixed this problem by focusing on the velocity fluctuations, performing HST simulations with only shear production and neglecting shear convection. The current study improves upon the previous simulations by including shear convection, by introducing an inflow/outflow in the cross-stream direction. To reduce the impact of the boundary conditions, an elongated domain is used. The simulation results show that the aspect ratio has very little effect on both isotropic and shear turbulence. When convection is included, the turbulence statistics still reach a statistically stationary state. The Reynolds shear stress and the anisotropy values agree very well with the results from experiments and simulations of mixing layers, planar jets, and round jets.
\end{abstract}

DOI: 10.1103/PhysRevFluids.5.124605

\section{INTRODUCTION}

Turbulent free shear flows are found in a multitude of industrial applications and in nature. However, owing to the range of scales and the stochastic and unsteady nature of turbulence, simulating such flows has proven to be quite challenging. Various configurations have been used to simulate turbulent flows using direct numerical simulations (DNS), which are resolved down to the smallest turbulent length scales.

A numerically efficient method to analyze the turbulence observed in the shear layers of practical free shear flows is to use homogeneous shear turbulence (HST) simulations. HST is characterized by a mean flow with a constant gradient perpendicular to the flow, while the turbulence statistics are homogeneous in all three directions. The turbulence in HST is dominated by two physical processes: shear production and shear convection. While shear production is homogeneous, shear convection involves the mean velocity and is nonhomogeneous. The nonhomogeneous shear convection term presents issues in HST simulations, as regular periodic boundary conditions cannot be used in the mean gradient direction. These issues have been tackled in past HST studies in different ways.

In 1981, Rogallo performed numerical HST experiments, using time-dependent coordinate transformations to maintain periodic boundary conditions, which meant the field had to be remeshed

*cdhandap@caltech.edu 
regularly [1]. The same was true in the case of Rogers and Moin's study [2] as well as for Lee et al. [3]. Baron [4] tackled the issue by introducing shear-periodic boundary conditions in the gradient direction, which was also implemented by Gerz et al. [5], Brucker et al. [6], Isaza and Collins et al. [7], and Kasbaoui et al. [8]. However, in all of these simulations, the turbulence statistics are not stationary [1-8].

Recently, Sekimoto et al. performed statistically stationary HST simulations for a wide range of aspect ratios. The study focused on the bursts in the time evolution of the turbulence statistics [9]. These bursts are not a result of the shear convection, as they were observed in simulations of isotropic turbulence [10] and of shear turbulence without shear convection [11]. Carroll and Blanquart were able to reduce these bursts by modifying the form of the linear forcing terms [10]. To date, Sekimoto et al. remains the only HST study with shear-periodic boundaries to achieve statistical stationarity. While Sekimoto et al. did achieve a statistically stationary state in their simulations, they did not investigate the structure, anisotropy, or scaling of the resulting turbulent field, nor did they compare them against the shear turbulence observed in practical flows. In fact, previous studies were focused on phenomenological shear in idealized HST flows and were not derived to match precise experimental configurations.

In contrast, Rah et al. [12] and Dhandapani et al. [11] performed simulations corresponding to particular locations in experimental configurations of turbulent flows, utilizing and extending the general linear forcing formalism used by Lundgren [13]. Rah et al. decomposed the instantaneous velocity, $\boldsymbol{u}$, into its mean, $\overline{\boldsymbol{u}}$, and fluctuating component, $\boldsymbol{u}^{\prime}$, at the centerline of a statistically stationary turbulent round jet. They captured the statistically stationary turbulence in a triply periodic cubic domain, by simulating just the velocity fluctuations [12]. The velocity anisotropy, energy spectrum, and turbulent kinetic energy budget from their simulations agreed well with experiments and full-domain DNS of turbulent round jets. It should be noted that the flow at the centerline has no shear convection. That is why the authors could use regular periodic boundary conditions.

Using similar techniques, the authors extended the study to simulate the shear layers of statistically stationary free shear flows, including turbulent mixing layers, turbulent planar jet, and round jets [11]. The study achieved the often elusive combination of homogeneity and statistical stationarity in HST calculations, by not including shear convection. The energy spectra from the simulations matched perfectly with experiments, and the anisotropy values show decent agreement with full-domain DNS and experiments, leaving a little room for improvement.

The current study improves upon the previous study by including shear convection in the simulation. Theoretically, shear convection does not impact the ensemble averaged TKE equation. However, no study has investigated the impact of shear convection separated from shear production, and its impact of the flow field has not been characterized. The goal of this study is threefold: (1) achieve statistically stationary turbulence with the inclusion of shear convection, without using shear-periodic boundary conditions, (2) compare the results with experiments of free shear flows, and (3) isolate the effects of shear convection and shear production. As mentioned earlier, as the mean velocity is nonhomogeneous, periodic boundary conditions cannot be used in the gradient direction. Instead of using shear-periodic boundaries or remeshing schemes (as done in previous studies), the objective is to use an inflow/outflow in the gradient direction. To avoid an influence of the boundary conditions on the turbulence statistics in the bulk of the domain, the domain width must be larger in the nonhomogeneous direction.

The numerical approach is presented in Sec. II followed by a stationary state analysis in Sec. III. The results are discussed in Sec. IV, where the effects of aspect ratio are detailed in Sec. IV A, the effects of inflow/outflow are discussed in Sec. IV B, and the effects of shear convection are analyzed in Sec. IV C. Finally, concluding remarks are presented in Sec. V. 


\section{NUMERICAL APPROACH}

\section{A. Governing equations}

The flows considered for the current study are incompressible turbulent flows, with constant density, $\rho$, and kinematic viscosity, $v$. This study follows the methodology of Lundgren [13], expanded to anisotropic flows by Rah et al. [12] and Dhandapani et al. [11]. Only a brief review of the methodology is provided here. The transport equation for the fluctuating velocity field, $\boldsymbol{u}^{\prime}=\boldsymbol{u}-\overline{\boldsymbol{u}}$, where ${ }^{-}$represents the ensemble average, is given by

$$
\frac{\partial \boldsymbol{u}^{\prime}}{\partial t}+\left(\overline{\boldsymbol{u}}+\boldsymbol{u}^{\prime}\right) \cdot \nabla \boldsymbol{u}^{\prime}=-\frac{1}{\rho} \nabla p^{\prime}+v \nabla^{2} \boldsymbol{u}^{\prime}+\nabla \cdot \overline{\boldsymbol{u}^{\prime} \boldsymbol{u}^{\prime}}-\boldsymbol{u}^{\prime} \cdot \nabla \overline{\boldsymbol{u}},
$$

where $p^{\prime}$ is the fluctuating pressure, $p^{\prime}=p-\bar{p}$. The linear production term, $-\boldsymbol{u}^{\prime} \cdot \nabla \overline{\boldsymbol{u}}$, and the advection by the mean flow, $-\overline{\boldsymbol{u}} \cdot \nabla \boldsymbol{u}$, significantly affect the turbulence statistics.

Equation (1) is rewritten in a mathematically equivalent form given by

$$
\frac{\partial \boldsymbol{u}^{\prime}}{\partial t}+\nabla \cdot\left(\boldsymbol{u}^{\prime} \otimes \boldsymbol{u}^{\prime}\right)=-\frac{\nabla p^{\prime}}{\rho}+v \nabla^{2} \boldsymbol{u}^{\prime}+\boldsymbol{f}-\boldsymbol{V} \cdot \nabla \boldsymbol{u}^{\prime},
$$

where $\boldsymbol{f}=-\boldsymbol{u}^{\prime} \cdot \nabla \overline{\boldsymbol{u}}$ is the linear forcing vector, which can be calculated based on the mean velocity gradients. For homogeneous isotropic turbulence (HIT), $\boldsymbol{f}=A \boldsymbol{u}^{\prime}$ [13]; at the centerline of a jet, $\boldsymbol{f}=$ $A \boldsymbol{u}^{\prime} / 2+A u_{y}^{\prime} / 2 \boldsymbol{e}_{\boldsymbol{y}}[12]$; and for shear-dominated regions in mixing layers, planar jets, and round jets, $\boldsymbol{f}=B u_{x}^{\prime} \boldsymbol{e}_{y}$ [11]. Note that the jet axis is aligned with the $y$ axis in this study. In the general case, the mean velocity gradient tensor, $-\nabla \overline{\boldsymbol{u}}$, would be a combination of diagonal elements and a dominant off-diagonal shear strain rate element. Hence, the forcing matrix containing only the diagonal elements and the forcing matrix with only the off-diagonal element can be seen as the two extreme cases of turbulence forcing. These two limiting cases (HIT and HST) will be explored further in the study.

The simulations are performed using NGA [14], a semi-implicit velocity solver with an energyconserving finite difference scheme on a standard staggered grid. The solver uses second-order schemes in space and for time integration. In all these simulations, the computational domains are meshed with a uniform grid, with equal spacing in the three directions, $\Delta x=\Delta y=\Delta z=L / 128$. The grid size is chosen such that $\Delta x \simeq 1.6 \eta_{k}$, where $\eta_{k}=\left(v^{3} / \epsilon\right)^{1 / 4}$ is the Kolomogorov length scale. This corresponds to $k_{\max } \eta \simeq 2.0$, where $k_{\max }$ is the highest resolved wave number. A value of $k_{\max } \eta>1.5$ is often reported as sufficient for numerical simulations of isotropic turbulence $[15,16]$. The same simulation framework was used by Desjardins et al. to perform HIT simulations using $k_{\max } \eta \simeq 1.5$, the spectra of which agreed perfectly with spectral simulations and experiments [14]. To ensure grid convergence, the authors performed doubly refined HST simulations in the $2 L \times L \times$ $L$ domains, and there were no differences in the statistics from the simulation results.

When discussing shear turbulence, the effect of shear convection on the turbulence statistics should also be analyzed. This requires including an imposed mean velocity in the simulation, which has a gradient of $-B$ in the cross-stream direction. This is not possible with a triply periodic computational domain, as the imposed mean velocity is not periodic. Hence, a new computational domain with inflow/outflow is required in order to include shear convection in these simulations. Domains with inflow/outflow need to be longer in that direction, so the boundary conditions do not affect the bulk of the domain.

\section{B. Flow configurations}

Simulations of increasing complexity are considered:

(1) Triply periodic, cubic domain

(2) Triply periodic, elongated domains

(3) Doubly periodic, elongated domain with inflow/outflow

(4) Doubly periodic, elongated domain with inflow/outflow and shear convection. 
TABLE I. Parameters of the triply periodic and inflow/outflow simulations.

\begin{tabular}{|c|c|c|c|c|c|c|c|c|c|c|c|}
\hline & \multicolumn{8}{|c|}{ Triply periodic } & & & \\
\hline & \multicolumn{2}{|c|}{ Cubic } & \multicolumn{6}{|c|}{ Long } & \multicolumn{3}{|c|}{ Inflow/outflow (I/O) } \\
\hline & Isotropic & Shear & Isotropic & Shear & Isotropi & Shear & Isotropic & Shear & Isotropic & Shear & Convection \\
\hline \multicolumn{12}{|c|}{ Domain } \\
\hline size & \multicolumn{2}{|c|}{$L \times L \times L$} & \multicolumn{2}{|c|}{$2 L \times L \times L$} & \multicolumn{2}{|c|}{$4 L \times L \times L$} & \multicolumn{2}{|c|}{$8 L \times L \times L$} & \multicolumn{3}{|c|}{$11 L \times L \times L$} \\
\hline Grid & \multicolumn{2}{|c|}{$128 \times 128 \times 128$} & \multicolumn{2}{|c|}{$256 \times 128 \times 128$} & \multicolumn{2}{|c|}{$512 \times 128 \times 128$} & \multicolumn{2}{|c|}{$1024 \times 128 \times 128$} & \multicolumn{3}{|c|}{$1408 \times 128 \times 128$} \\
\hline$f$ & $A u^{\prime}$ & $B u_{x}^{\prime} \boldsymbol{e}_{\boldsymbol{y}}$ & $A \boldsymbol{u}^{\prime}$ & $B u_{x}^{\prime} \boldsymbol{e}_{\boldsymbol{y}}$ & $A \boldsymbol{u}^{\prime}$ & $B u_{x}^{\prime} \boldsymbol{e}_{\boldsymbol{y}}$ & $A \boldsymbol{u}^{\prime}$ & $B u_{x}^{\prime} \boldsymbol{e}_{\boldsymbol{y}}$ & $A \boldsymbol{u}^{\prime}$ & $B u_{x}^{\prime} \boldsymbol{e}_{\boldsymbol{y}}$ & $B u_{x}^{\prime} \boldsymbol{e}_{\boldsymbol{y}}$ \\
\hline$V$ & 0 & 0 & 0 & 0 & 0 & 0 & 0 & 0 & 0 & 0 & $V e_{y}$ \\
\hline $\operatorname{Re}_{t}$ & 160 & 150 & 145 & 150 & 140 & 150 & 130 & 150 & 130 & 130 & 210 \\
\hline $\operatorname{Re}_{\lambda}$ & 50 & 50 & 50 & 50 & 45 & 50 & 45 & 50 & 45 & 45 & 55 \\
\hline $\bar{\ell} / L$ & 0.18 & 0.25 & 0.17 & 0.25 & 0.17 & 0.25 & 0.16 & 0.25 & 0.16 & 0.23 & 0.33 \\
\hline $\bar{\beta}$ & -0.03 & 0.40 & 0.00 & 0.39 & 0.00 & 0.38 & 0.00 & 0.38 & 0.00 & 0.41 & 0.31 \\
\hline
\end{tabular}

The simulation parameters for the different simulations are presented in Table I. The domain width, viscosity, and forcing constant are kept constant between the simulations, so the effects of the aspect ratio, inflow/outflow, and shear convection on the turbulent flow can be isolated and analyzed.

\section{Triply periodic domain}

All simulations in the current section rely on periodic boundary conditions and result in statistical homogeneity in all three directions. Therefore, ensemble averages can be replaced by volume averages, calculated as

$$
\langle a\rangle(t)=\frac{1}{L^{2} L_{x}} \int_{x} \int_{y} \int_{z} a(x, y, z, t) d x d y d z
$$

where $L_{x}$ is the domain width in the $x$ direction. Isotropic and shear turbulence simulations are performed in four domains with different aspect ratios. The first one is a cubic domain with a width of $L$ in each direction, $L_{x}=L$. Each subsequent domain is doubled in length in the $x$ direction, $L_{x}=2 L, 4 L, 8 L$.

The simulations are initialized with velocity fields from the results of previously performed HST simulations, at similar turbulence parameters [11]. The velocity fields are copied over multiple times for the initial conditions of the long domain simulations, and random small perturbations (of $1 \%$ magnitude compared to $u_{\mathrm{rms}}$ ) in velocity are included to break symmetry. The time evolution of the long domain simulation statistics deviate quickly from that of cubic domain simulations, and symmetry is broken within $5 \tau_{0}$.

\section{Domain with inflow and outflow}

The simulations utilize the same flow configuration used in previous work to simulate turbulent flames [17-21]. The computational domain is doubly periodic in the $y$ and $z$ directions, and has an inflow/outflow in the $x$ direction. The velocity fields are subjected to the forcing term, $f$, multiplied by a proportionality function, $\alpha(x)$ which is unity between $L$ and $9 L$. This proportionality function is shown in Fig. 1. The turbulence forcing is maintained at zero at the inlet to reduce instances of local outflow and at the outlet to reduce instances of local inflow. Near the outflow, the flow needs the larger buffer distance to enable the decay of turbulence.

Three different simulations are performed: one with isotropic turbulence, one with shear turbulence, and one with both shear forcing and shear convection.

Leveraging the long domain and the inflow/outflow boundary conditions in the $x$ direction, shear convection is applied in a portion of the computational domain. More specifically, we introduce a 


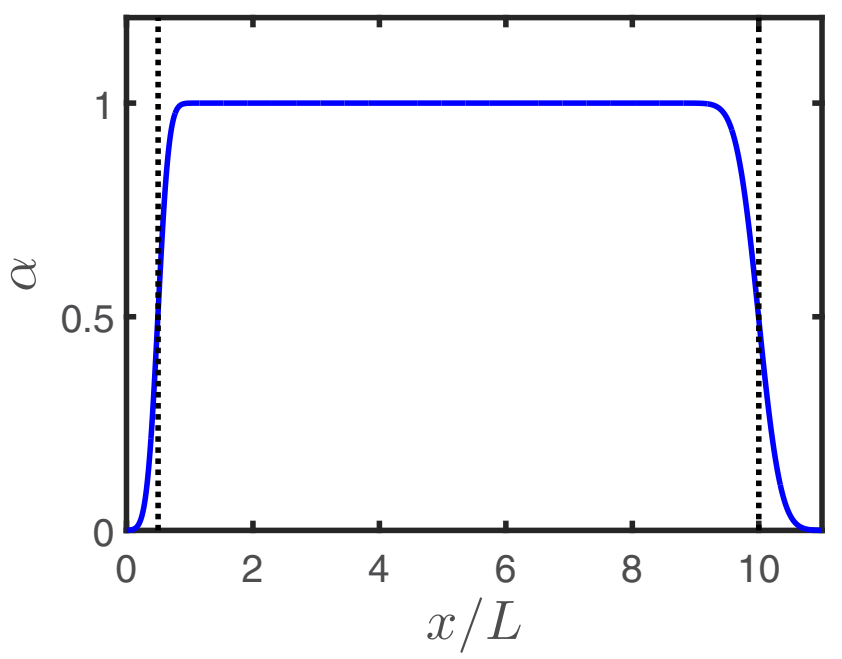

FIG. 1. Proportionality function profile.

convection velocity, $\boldsymbol{V}=V \boldsymbol{e}_{\boldsymbol{y}}$, which has a gradient of $-B$ in a portion of the domain between $3.5 \mathrm{~L}$ and $7.5 \mathrm{~L}$ and $V$ is constant in the rest of the domain. A profile of the convection velocity as a function of $x$, normalized by the domain width and shear forcing constant, is shown in Fig. 2.

The portion with a mean velocity gradient of $-B$ corresponds to homogeneous shear turbulence with shear convection, whereas the two portions with constant $V$ correspond to the shear simulation with no shear convection. The shear convection is applied to a region approximately a third of the domain, so the region with shear convection can be directly compared to the regions without, in the same simulation.

Given the statistical homogeneity along the $y$ and $z$ directions, ensemble averages can be replaced by planar averages, calculated as

$$
\langle a\rangle(x, t)=\frac{1}{L^{2}} \int_{y} \int_{z} a(x, y, z, t) d y d z
$$

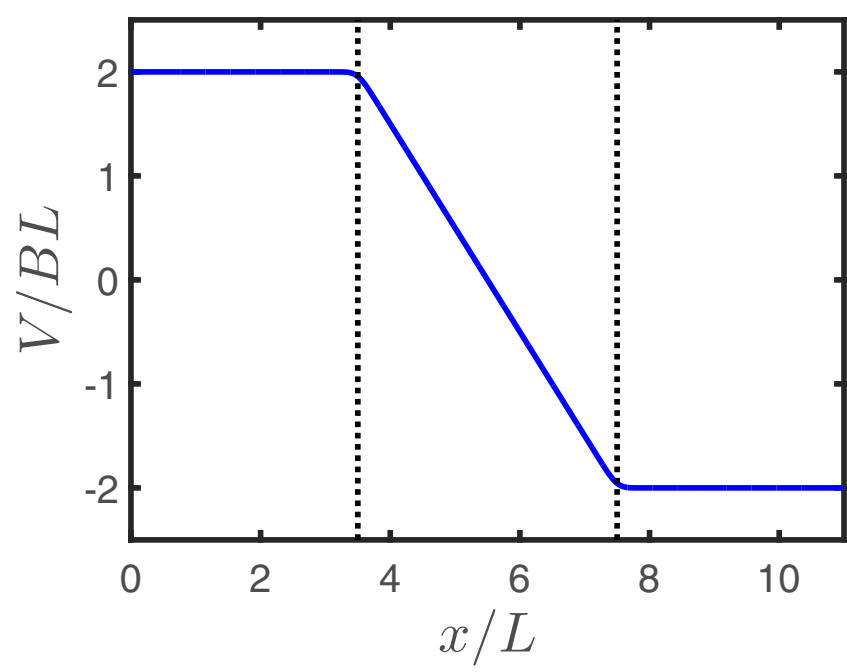

FIG. 2. Convection velocity profile normalized by the shear forcing constant and domain width. 
The mean velocity, $\bar{u}$, of the inflow is chosen to be constant in time. Superimposed on the mean velocity at the inflow are fields of fluctuating velocities computed from simulations of homogeneous isotropic turbulence at a lower turbulent Reynolds number. The turbulent kinetic energy at the outlet must be small enough to not have any instances of local inflow, hence a large enough buffer is needed over which the turbulence can dissipate. The mean velocity has to be small enough such that the Lagrangian particles take a long enough time to travel over this buffer region. The chosen mean velocity is slightly larger than the rms velocity at the inlet, $\bar{u} \sim 1.15 u_{\mathrm{rms}}$ and the forcing is stopped at $10 \mathrm{~L}$. The time taken by Lagrangian particles to travel a distance of $L$ in the $x$ direction is $20 \tau_{o}$. This ensures that the flow is close to laminar at the outlet, and convective outflow boundary condition is used. At the inlet, Dirichlet boundary condition is used. There are few instances of local outflow at the inlet, but it has no effect on either the stability of the simulation or the turbulence statistics within the domain.

\section{STATIONARY STATE ANALYSIS}

By construction, the velocity field represents the fluctuations of the flow field in a small region of a statistically stationary turbulent flow. Hence, the fluctuating quantities and their related statistics must reach a statistically stationary state. This applies to turbulent kinetic energy, dissipation rate, Reynolds stress, etc. We briefly review the scaling found in Carroll and Blanquart [10] for HIT and Dhandapani et al. [11] for HST.

\section{A. HIT in cubic domain}

The turbulent kinetic energy equation for the isotropic turbulence forcing, assuming spatial homogeneity, is

$$
\frac{d k}{d t}=-\varepsilon+2 A k
$$

At statistically stationary state, the energy dissipation rate is

$$
\varepsilon=2 A k \text {. }
$$

The integral length scale, $\ell$, is defined as

$$
\ell=\frac{u_{\mathrm{rms}}^{3}}{\varepsilon}=\frac{u_{\mathrm{rms}}}{3 A},
$$

with $u_{\mathrm{rms}}=\sqrt{2 k / 3}=3 \mathrm{~A} \ell$.

The expected turbulent Reynolds number is calculated as

$$
\operatorname{Re}_{t, i}^{o}=\frac{u_{\mathrm{rms}} \ell}{v}=\frac{3 A \ell^{2}}{v},
$$

The Taylor microscale, $\lambda$, is calculated as

$$
\lambda=u_{\mathrm{rms}} \sqrt{15 \frac{\nu}{\varepsilon}}
$$

The expected Taylor microscale Reynolds number is given by

$$
\operatorname{Re}_{\lambda, i}^{o}=\sqrt{\frac{45 A \ell^{2}}{v}},
$$

and the expected values for turbulent kinetic energy, $k_{o, i}$, and energy dissipation rate, $\varepsilon_{o, i}$, can be calculated as

$$
k_{o, i}=\frac{3}{2} u_{\mathrm{rms}}^{2}=\frac{27}{2} A^{2} \ell^{2}
$$


and

$$
\varepsilon_{o, i}=\frac{u_{\mathrm{rms}}^{3}}{\ell}=27 A^{3} \ell^{2}
$$

The expected eddy turnover time $\tau_{o, i}$ is given by

$$
\tau_{o, i}=\frac{k_{o, i}}{\varepsilon_{o, i}}=\frac{1}{2 A} .
$$

Finally, the ratio of integral length scale to domain size, was found to be $\ell / L \simeq 0.19$ for isotropic turbulence in a triply periodic box domain [10,22]. This value was found to be independent of the Reynolds number.

\section{B. HST without shear convection in cubic domain}

It was shown by Dhandapani et al. [11] that for HST without shear convection, the turbulent kinetic energy equation is

$$
\frac{d k}{d t}=-\varepsilon+B\left\langle u_{x}^{\prime} u_{y}^{\prime}\right\rangle .
$$

At statistically stationary state, the energy dissipation rate is

$$
\varepsilon=B\left\langle u_{x}^{\prime} u_{y}^{\prime}\right\rangle=B \beta k,
$$

with $u_{\mathrm{rms}}=3 B \beta \ell / 2$. The expected values for turbulent kinetic energy, $k_{o, s}$, and energy dissipation rate, $\varepsilon_{o, s}$, can be calculated as

$$
k_{o, s}=\frac{27}{8} \beta^{2} B^{2} \ell^{2}
$$

and

$$
\varepsilon_{o, s}=\frac{27}{8} \beta^{3} B^{3} \ell^{2}
$$

The expected turbulent Reynolds number is calculated as

$$
\operatorname{Re}_{t, s}^{o}=\frac{3 B \beta \ell^{2}}{2 v}
$$

and the expected eddy turnover time, $\tau_{o}$, is given by

$$
\tau_{o, s}=\frac{1}{\beta B} \text {. }
$$

It is ideal for the isotropic and shear simulations to have similar turbulent Reynolds numbers. The relationship between the two forcing constants can be calculated by equating the two expected turbulent Reynolds numbers:

$$
\operatorname{Re}_{t, i}^{o}=\operatorname{Re}_{t, s}^{o} \Rightarrow B=\frac{2 A}{\beta}\left(\frac{\ell_{i}}{\ell_{s}}\right)^{2}
$$

\section{RESULTS}

The results are decomposed into three groups: the effects of aspect ratio, the effects of inflow/outflow, and the effects of shear convection. 


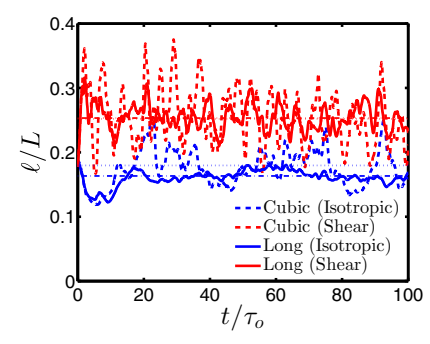

(a) Statistical stationarity

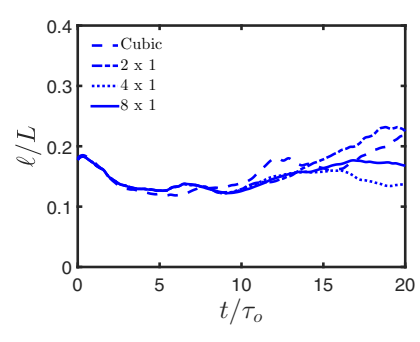

(b) Short term evolution

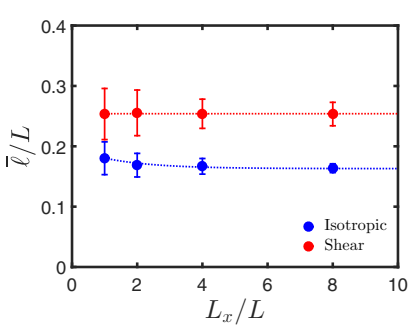

(c) Impact of aspect ratio

FIG. 3. Integral length scale normalized by the domain width $L$, from the triply periodic simulations using isotropic (blue) and shear (red) turbulence. Time evolution ( $a$ and b) for cubic (dashed lines) and long domains (solid lines: $8 L$, dotted lines: $4 L$, dashed dot lines: $2 L$ ). (c) Average integral length scale plotted against the aspect ratio. Blue dotted line in (c) corresponds to an exponential fit, and red dotted line represents the average value.

\section{A. Effects of aspect ratio}

The effects of the aspect ratio on the turbulence quantities are analyzed by observing the differences between the cubic and long domain simulations for both isotropic and shear turbulence. All eight triply periodic simulations are performed for 100 eddy turnover times and averages are calculated after a transient period of $20 \tau_{0}$. The effects of the aspect ratio on the turbulence and flow anisotropy are discussed in the following subsections.

\section{Isotropic turbulence}

The time evolution of the normalized integral length scale is shown in Figs. 3(a) and 3(b). The volume averages from the long domain simulation have much smaller oscillations owing to better statistical convergence due to the larger number of data points. In statistically stationary, grid-converged turbulence, the uncertainties in statistical averages are inversely proportional to the square root of the number of individual realizations, hence the uncertainties in the $8 L$ case would be $\sqrt{8} \sim 2.8$ times smaller than the uncertainties in the cubic case. The oscillations in the cubic simulation results are well known and can theoretically be reduced by using a modification proposed by Carroll and Blanquart [10], but the current study does not use the modification for a cleaner comparison between cases.

As mentioned earlier, random perturbations are introduced in the initial data files to break the symmetry between the simulations. The integral length scales for the long domain simulations deviate from that of the cubic domain in less than $5 \tau_{0}$ and from each other in about $10 \tau_{0}$. Statistical stationarity is observed at about $20 \tau_{0}$. The average integral length scale is calculated as $\bar{\ell}=\frac{1}{80 \tau_{o}} \int_{20 \tau_{o}}^{100 \tau_{o}} u_{\mathrm{rms}}(t)^{3} / \varepsilon(t) d t$ and has a weak dependence on the aspect ratio as seen in Fig. 3(c). While the cubic simulation has an average integral length scale value of about $\bar{\ell} \simeq 0.18 \mathrm{~L}$, the longest domain case $(8 L)$ shows a smaller integral length scale of about $\bar{\ell} \simeq 0.16 L$. Similar reductions are seen in the average integral length scales for the $2 L(\bar{\ell} \simeq 0.17 L)$ and $4 L(\bar{\ell} \simeq 0.17 L)$ cases. The integral length scales in the long domains remain controlled by the smallest dimension length, $L$, (and not $L_{x}$ ), and the reduced integral length scales are consistent with the observations of the turbulent reacting flow simulations performed by Brock et al. [17]. This difference explains the lower Reynolds number, as it is directly proportional to the square of the integral length scale [Eq. (8)].

The time evolution of the turbulent kinetic energy is also plotted for the cubic and long domain isotropic cases in Fig. 4(b), normalized by their respective expected values, $k_{o, i}$, calculated using their respective integral length scale values, and they fluctuate around a value of 1 . Bursts in kinetic energy are observed in the time evolution, which are more prominent in the cubic domain 


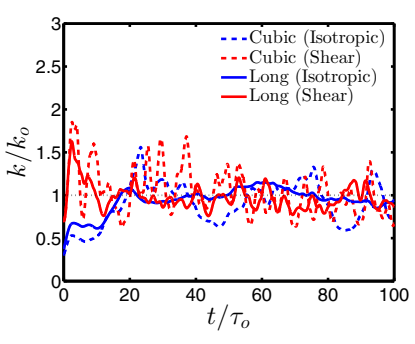

(a) Turbulent kinetic energy

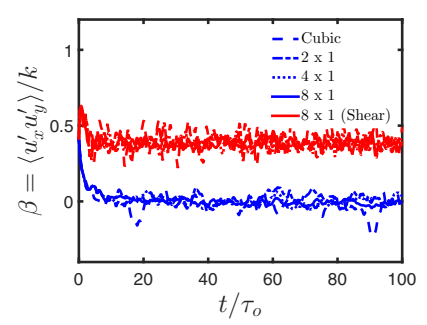

(b) Reynolds shear stress

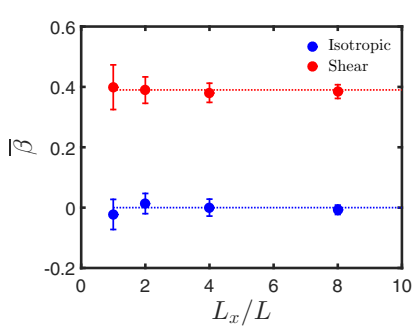

(c) Average shear stress

FIG. 4. Triply periodic simulation results using isotropic (blue) and shear (red) turbulence. Time evolution of the turbulent kinetic energy normalized by the expected values, $k_{o}$ (a) and the Reynolds shear stress normalized by the turbulent kinetic energy (b) for cubic (dashed lines) and long domains (solid lines: $8 L$, dotted lines: $4 L$, dashed dot lines: $2 L$ ). (c) Average Reynolds shear stress normalized by the turbulent kinetic energy, plotted against the aspect ratio. The dotted lines represent the average values.

simulation. These bursts in turbulence were documented by Carroll and Blanquart, who modified the linear forcing term, to make the simulations more stable in time [10].

\section{Shear turbulence}

The evolution of the normalized integral length scale is shown in Fig. 3(a). Statistical stationarity is reached quickly, within $20 \tau_{0}$, and the integral length scales for each case fluctuate around a constant value. Figure 3(c) shows the temporal averages of the integral length scale computed as in the isotropic case. Unlike for isotropic turbulence, there is no change in integral length scale in longer domains for shear turbulence. The time evolution of the volume-averaged kinetic energy is plotted for the cubic and long domain $(8 L)$ shear cases in Fig. 4(b), normalized by their expected values, $k_{o, s}$. The two simulations have similar initial profile, but the long domain has much smaller oscillations owing (once again) to the larger number of data points, and the results from both cases fluctuate around the expected values. The bursts in kinetic energy appear more often compared to isotropic turbulence simulations, and are stronger in the cubic domain simulation. Sekimoto et al. found bursts in the turbulence statistics in shear turbulence simulations. These bursts were observed to have a timescale of the order of $20 B^{-1}$ (corresponds to $8 \tau_{o}$ ) [9], which is consistent with the timescale found in Fig. 4(b). This characteristic timescale is calculated from the number of peaks in the time signal in a large enough time period. The timescale will not be revealed by frequency analysis, as these bursts are random events and do not have a single characteristic frequency.

The time evolution of the Reynolds shear stress normalized by the turbulent kinetic energy is plotted in Fig. 4(b), for all eight simulations. The average value of $\beta$, calculated as $\bar{\beta}=$ $\frac{1}{80 \tau_{o}} \int_{20 \tau_{o}}^{100 \tau_{o}}\left\langle u_{x}^{\prime} u_{y}^{\prime}\right\rangle(t) / k(t) d t$, is $0.40 \pm 0.07$ for the cubic simulation and 0.38 to 0.39 for the long domain simulations. The Reynolds shear stress is not affected by the aspect ratio of the computational domain, as seen in Fig. 4(c).

\section{B. Effects of inflow/outflow}

The effects of the inflow/outflow on the turbulence quantities are analyzed by observing the differences between the triply periodic long domain simulations and the inflow/outflow simulations for isotropic and shear turbulence.

\section{Global quantities}

The time averaged integral length scale profile is plotted for the inflow/outflow cases in Fig. 5(a). The integral length scale is $\bar{\ell} \simeq 0.16 L$ for the isotropic forcing and is the same value as for the triply periodic long domain. The value is $\bar{\ell} \simeq 0.23 L$ for the shear forcing, very close to the 0.25 


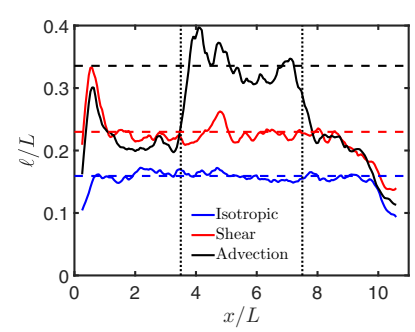

(a) Integral length scale

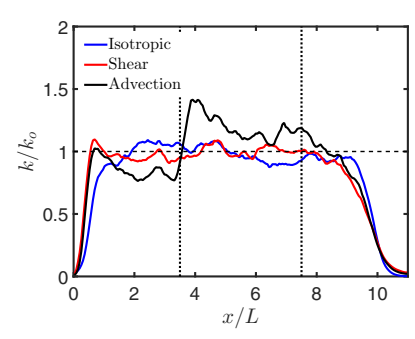

(b) Turbulent kinetic energy

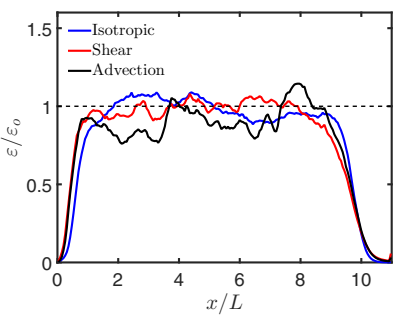

(c) Energy dissipation rate

FIG. 5. Inflow/outflow simulation results: Planar and temporal averages of the (a) integral length scale normalized by the domain width, (b) turbulent kinetic energy profile normalized by the expected value, $k_{o}$, and (c) energy dissipation rate normalized by the expected value, $\varepsilon_{o}$, for isotropic (blue), shear (red), and convection (black) cases. Dotted lines correspond to the edges of the region where shear convection is included. Dashed lines in (a) correspond to average values calculated between $L$ and $9 L$ for the isotropic and shear cases, and $4 L$ to $7 L$ for the advection case.

value found for triply periodic long domain, and is about 1.5 times the integral length scale for the isotropic case. These values are used in the calculation of the expected values of the turbulent kinetic energy and the dissipation rate.

The time-averaged kinetic energy and energy dissipation rate profiles are plotted over the domain for the two cases in Fig. 5, normalized by their respective expected values. The two simulations have similar kinetic energy and energy dissipation rate profiles as seen in Fig. 5. The values are much lower near the inlet/outlet, where the forcing is not applied and have small fluctuations around the expected values for most of the domain, between $L$ and $9 L$. The region with homogeneous turbulence statistics has a domain width of $8 L$ and is comparable to the triply periodic elongated domain simulations $\left(L_{x}=8 L\right)$. The volume averages calculated between $L$ and $9 L$ are observed to be statistically stationary after $20 \tau_{0}$, just like in the triply periodic simulations, as seen in Fig. 6 . The volume average of the turbulent kinetic energy calculated between $L$ and $9 L$ fluctuates around the expected value over long time periods, signaling the simulations are statistically stationary.

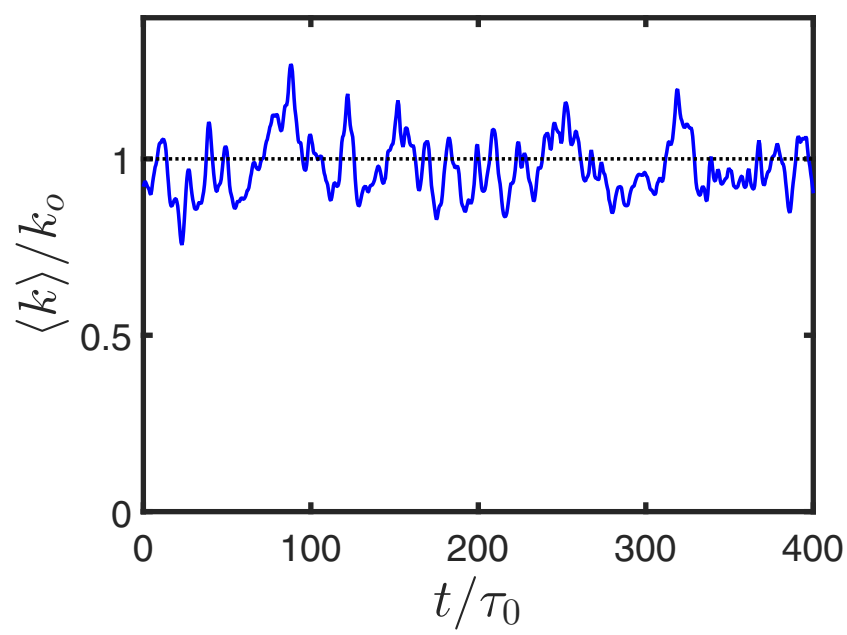

FIG. 6. Time evolution of the volume average of the turbulent kinetic energy from the inflow/outflow simulation with isotropic forcing, calculated between $L$ and $9 L$. 


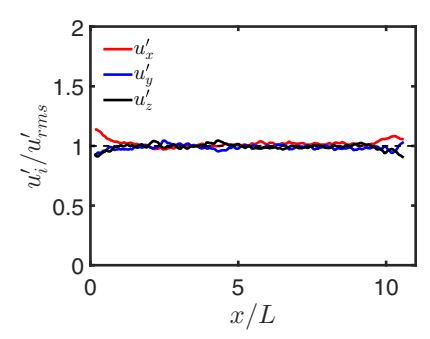

(a) $u^{\prime}$ (isotropic)

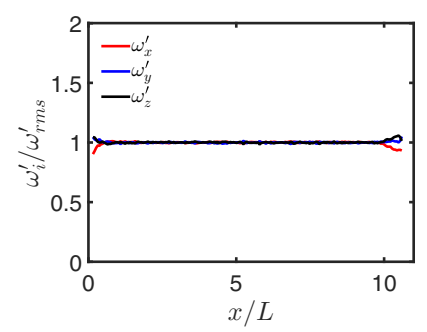

(d) $\omega^{\prime}$ (isotropic)

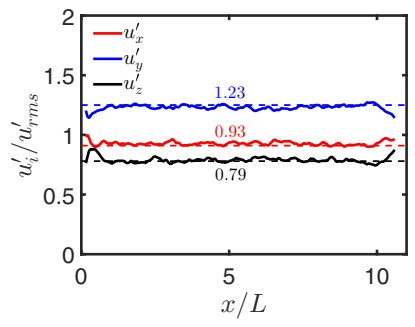

(b) $u^{\prime}$ (shear)

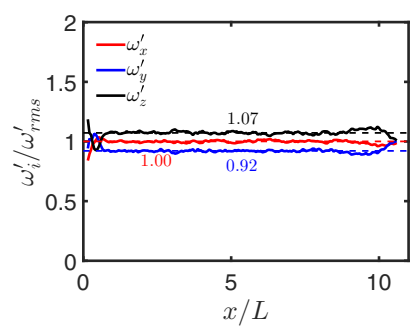

(e) $\omega^{\prime}$ (shear)

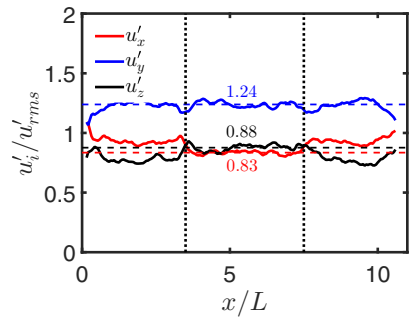

(c) $u^{\prime}($ convection)

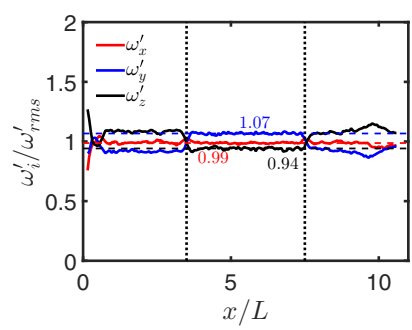

(f) $\omega^{\prime}$ (convection)

FIG. 7. Velocity fluctuation magnitudes for the isotropic (a), shear (b), and convection (c), normalized by the root mean square velocity, $u_{\mathrm{rms}}$. Vorticity fluctuation magnitudes normalized by the root mean square velocity, $\omega_{\text {rms }}$, for the isotropic (d), shear (e), and convection (f) cases. Dotted lines in (c) and (f) correspond to the edges of the region where shear convection is included. Dashed lines in (b) correspond to average values from the triply periodic long domain simulations and in (c) correspond to average values calculated between $4 L$ and $7 L$.

\section{Flow anisotropy}

As shown in Sec. IV B 1, the turbulence intensity is comparable between the different forcing techniques, but large differences are expected in the turbulence anisotropy. This anisotropy can be characterized by examining the components of the velocity and vorticity vectors.

The magnitudes of the fluctuating velocity components, $\left|u_{i}^{\prime}\right|=\sqrt{\left\langle u_{i}^{\prime 2}\right\rangle}$, are plotted as a function of space, normalized by $u_{\text {rms }}=\sqrt{\langle\boldsymbol{u} \cdot \boldsymbol{u}\rangle / 3}$ in Fig. 7(a) for the isotropic forcing and Fig. 7(b) for the shear forcing. For the isotropic forcing, the velocity fluctuations are statistically isotropic throughout, as with the triply periodic simulations. With shear forcing, the velocity components are significantly anisotropic throughout the domain. It is important to note that for the shear forcing case, the shear forcing is applied in the $y$ direction, proportional to the velocity fluctuations in the $x$ direction. The velocity components are the strongest in magnitude in the forced direction $(y)$, which is consistent with simulations from Dhandapani et al. [11]. The average values of the velocity anisotropy are calculated as $\left|u_{x}^{\prime}\right| / u_{\mathrm{rms}}=0.93,\left|u_{y}^{\prime}\right| / u_{\mathrm{rms}}=1.23$, and $\left|u_{z}^{\prime}\right| / u_{\mathrm{rms}}=0.79$, compared to the values from the triply periodic elongated domains given by $0.90,1.25$, and 0.78 , respectively, whereas the results from the triply periodic cubic domains are $0.91,1.24$, and 0.79 . Clearly, the aspect ratio and the inflow/outflow do not affect the anisotropy in velocity, within the range of parameters considered in this study.

The anisotropy in the small scales can be studied by evaluating the rms vorticity components along the different directions, $\left|\omega_{i}^{\prime}\right|=\sqrt{\left\langle\omega_{i}^{\prime 2}\right\rangle}$. They are shown, normalized by $\omega_{\mathrm{rms}}=\sqrt{\langle\boldsymbol{\omega} \cdot \boldsymbol{\omega}\rangle / 3}$ for the isotropic forcing in Fig. 7(d) and for the shear forcing in Fig. 7(e). The isotropic forcing technique produces isotropic vorticity fields $\left|\omega_{i}^{\prime}\right| / \omega_{\text {rms }} \simeq 1$ throughout the domain. In contrast and as mentioned previously, in the shear forcing case, the vorticity is somewhat anisotropic. The vorticity 


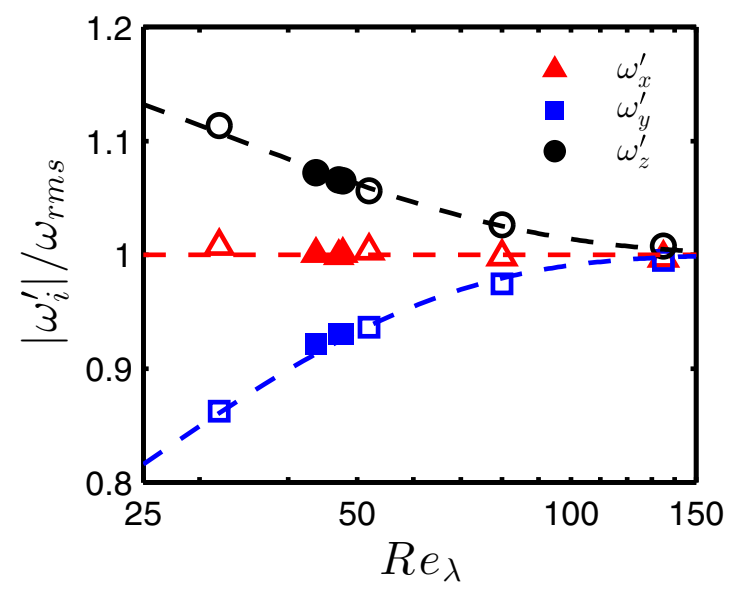

FIG. 8. Vorticity values calculated from the present shear turbulence simulations without shear convection (closed symbols) and from previously performed HST simulations [11] (open symbols), with exponential fits (dashed lines) calculated based on results from Dhandapani et al. [11].

component magnitudes are given by $\left|\omega_{x}^{\prime}\right| / \omega_{\text {rms }}=1.00,\left|\omega_{y}^{\prime}\right| / \omega_{\text {rms }}=1.07$, and $\left|\omega_{z}^{\prime}\right| / \omega_{\text {rms }}=0.92$. Dhandapani et al. [11] observed that the vorticity becomes more isotropic with increasing Reynolds number in HST without shear convection. Their average vorticity anisotropy values are plotted against Reynolds number in Fig. 8, along with exponential fits to those values. The vorticity anisotropy values of the present three shear turbulence simulations without shear convection (cubic, long, and inflow/outflow) are also plotted in the figure. The values are consistent with the exponential fits, based on results from previously performed HST simulations in triply periodic cubic domains.

Another significant measure of the anisotropy of the flow is the Reynolds shear stress. Figure 9(b) shows the Reynolds shear stress profile normalized by the turbulent kinetic energy for the isotropic and shear case. As expected, there is zero Reynolds shear stress in the isotropic case, as there is no cross-correlation of the velocity components. For the shear case, however the Reynolds shear stress is positive, and has a value of $\bar{\beta}=0.41$, which agrees well with results from triply periodic simulations $(0.40 \pm 0.06$ for cubic and $0.38 \pm 0.02$ for long).

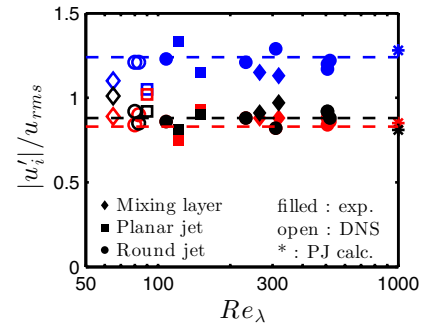

(a) Anisotropy in $u^{\prime}$

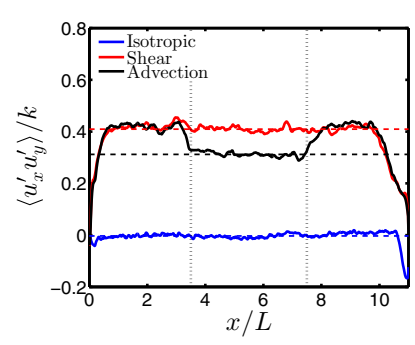

(b) Reynolds shear stress

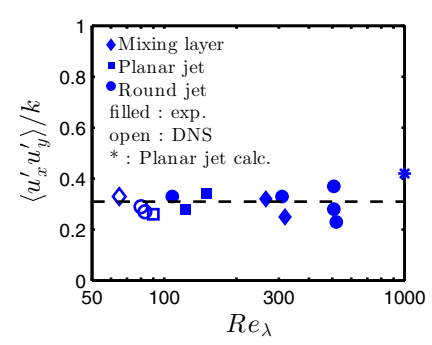

(c) Reynolds shear stress

FIG. 9. (a) Anisotropy in velocity from published studies of free shear flows as a function of Reynolds number for $u_{x}^{\prime}$ (red), $u_{y}^{\prime}$ (blue), and $u_{z}^{\prime}$ (black). (b) Reynolds shear stress profile normalized by the turbulent kinetic energy for isotropic (blue), shear (red), and convection (black) cases. Dashed lines correspond to average values. (c) Reynolds shear stress values as a function of Reynolds number. The dashed lines in (a) and (c) correspond to the values from the portion with the shear convection included from the convection case. See Ref. [11] for the complete list and details of the studies. 


\section{Effects of shear convection}

As mentioned previously, the shear convection velocity in the simulation, $\boldsymbol{V}$, is in the $y$ direction, and has a gradient of $-B$ from $x=3.5 L$ to $x=7.5 L$, in the middle of the domain. This portion is used to compare and contrast the turbulence statistics between simulations with just shear turbulence production, and simulations with shear turbulence production and shear convection.

\section{Global quantities}

The integral length scale is shown in Fig. 5(a). As expected, the integral length scale is different for all three simulations in the central region of the domain. The average integral length scale is calculated between $x=4 L$ to $x=7 L$ for the convection case. The results show that $\bar{\ell} \simeq 0.23 L$ for the shear case and $\bar{\ell} \simeq 0.33 L$ when shear convection is included. In other words, the integral length scale is larger when shear convection is included, by about $50 \%$. The time-averaged kinetic energy and energy dissipation rate profiles are plotted over the domain for the three cases in Fig. 5, normalized by their respective expected values. The three simulations have similar kinetic energy and energy dissipation rate as seen in Fig. 5 outside the convection region, but the turbulent kinetic energy is once again much higher in the region with shear convection, and the dissipation rate is slightly lower. Note that the profiles for the convection case are normalized by the expected values of the shear case without shear convection, $k_{o, s}$ and $\varepsilon_{o, s}$.

As shown from the results, when shear convection is included, $\bar{\ell} / L \simeq 0.33$ and $\bar{\beta} \simeq 0.31$. So the expected turbulence quantities with convection can be calculated as

$$
\begin{aligned}
& k_{o, a}=k_{o, s}\left(\frac{\beta_{a}}{\beta_{s}}\right)^{2}\left(\frac{\ell_{a}}{\ell_{s}}\right)^{2} \simeq 1.21 k_{o, s}, \\
& \varepsilon_{o, a}=\varepsilon_{o, s}\left(\frac{\beta_{a}}{\beta_{s}}\right)^{3}\left(\frac{\ell_{a}}{\ell_{s}}\right)^{2} \simeq 0.92 \varepsilon_{o, s},
\end{aligned}
$$

and

$$
\operatorname{Re}_{t, a}^{o}=\operatorname{Re}_{t, s}^{o}\left(\frac{\beta_{a}}{\beta_{s}}\right)\left(\frac{\ell_{a}}{\ell_{s}}\right)^{2} \simeq 1.60 \operatorname{Re}_{t, s}^{o} .
$$

This explains the higher Reynolds number and turbulent kinetic energy and slightly lower dissipation observed where shear convection is included.

\section{Flow anisotropy}

The anisotropy is once again characterized by examining the components of the velocity and vorticity vectors, specifically in the region between $3.5 \mathrm{~L}$ and $7.5 \mathrm{~L}$.

The magnitudes of the fluctuating velocity components are plotted, normalized by $u_{\mathrm{rms}}$ in Fig. 7(b) for the shear case and Fig. 7(c) for the convection case. With shear forcing, the velocity components are anisotropic throughout the domain, and their values are given by $\left|u_{x}^{\prime}\right| / u_{\mathrm{rms}}=0.93$, $\left|u_{y}^{\prime}\right| / u_{\mathrm{rms}}=1.23$, and $\left|u_{z}^{\prime}\right| / u_{\mathrm{rms}}=0.79$. In the portion where shear convection is included, the velocity components are still anisotropic, but the values are slightly different, and now $\left|u_{x}^{\prime}\right|$ is the weakest, with the values given by $\left|u_{x}^{\prime}\right| / u_{\mathrm{rms}}=0.83,\left|u_{y}^{\prime}\right| / u_{\mathrm{rms}}=1.24$, and $\left|u_{z}^{\prime}\right| / u_{\mathrm{rms}}=0.88$. These values agree better with experiments and simulations of shear-dominated flows like mixing layers and jets, as seen in Fig. 9(a). It is important to note that since the forcing is applied in the $y$ direction proportional to the fluctuations in the $x$ directions, $u_{y}^{\prime}$ should be compared to the streamwise velocity fluctuations and $u_{x}^{\prime}$ should be compared to the cross-stream velocity fluctuations. The experimental results show no evident dependence on Reynolds number, and the results from our simulation lie in the middle of the range of those values. In contrast, the study by Sekimoto et al. provides a wide range of values dependent on the aspect ratio of the computational domain.

The rms vorticity components along the different directions, $\left|\omega_{i}^{\prime}\right|$, normalized by $\omega_{\text {rms }}$ are plotted for the shear simulation in Fig. 7(e) and for the convection case in Fig. 7(f). The shear simulation 
TABLE II. Third- and fourth-order moments of velocity fluctuations calculated from the inflow/outflow simulations and compared with experimental results. DNS $3 \mathrm{~b}$ was a shear turbulence simulation in a triply periodic cubic domain $\left(192^{3}\right)$, with linear and nonlinear forcing terms.

\begin{tabular}{|c|c|c|c|c|c|c|}
\hline & $\overline{\left\langle u_{x}^{\prime 3}\right\rangle /\left\langle u_{x}^{\prime 2}\right\rangle^{3 /}}$ & $\sqrt{2} \overline{\left\langle u_{y}^{\prime 3}\right\rangle}$ & $3\rangle /\left\langle u_{y}^{\prime 2}\right\rangle^{3 / 2}$ & $\overline{\left\langle u_{x}^{\prime} u_{y}^{\prime 2}\right\rangle /\left\langle u_{y}^{\prime 2}\right\rangle\left\langle u_{x}^{\prime 2}\right\rangle^{1 / 2}}$ & $\overline{\left\langle u_{x}^{\prime 2} u_{y}^{\prime}\right\rangle /\left\langle u_{x}^{\prime 2}\right\rangle\left\langle u_{y}^{\prime 2}\right\rangle^{1 / 2}}$ & $\overline{\left\langle u_{y}^{\prime} u_{z}^{\prime 2}\right\rangle /\left\langle u_{z}^{\prime 2}\right\rangle\left\langle u_{y}^{\prime 2}\right\rangle^{1 / 2}}$ \\
\hline Isotropic & 0.02 & & -0.01 & 0.01 & 0.01 & 0.00 \\
\hline Shear & -0.13 & & 0.09 & 0.02 & -0.05 & -0.07 \\
\hline Convection & -0.10 & & 0.05 & 0.01 & -0.02 & -0.01 \\
\hline DNS 3b [11] & 0.26 & & 0.41 & - & - & - \\
\hline \multirow{2}{*}{$\begin{array}{l}\text { Panchapakesan } \\
\text { and Lumley [23] } \\
\text { Hussein } \text { et al. [24] }\end{array}$} & 0.39 & & 0.44 & 0.21 & 0.17 & 0.26 \\
\hline & 0.45 & & 0.37 & 0.27 & 0.26 & 0.19 \\
\hline \multicolumn{7}{|c|}{$\overline{\left\langle u_{x}^{\prime 4}\right\rangle /\left\langle u_{x}^{\prime 2}\right\rangle^{2}} \overline{\left\langle u_{y}^{\prime 4}\right\rangle /\left\langle u_{y}^{\prime 2}\right\rangle^{2}} \overline{\left\langle u_{z}^{\prime 4}\right\rangle /\left\langle u_{z}^{\prime 2}\right\rangle^{2}} \overline{\left\langle u_{x}^{\prime 2} u_{y}^{\prime 2}\right\rangle /\left\langle u_{x}^{\prime 2}\right\rangle\left\langle u_{y}^{\prime 2}\right\rangle} \overline{\left\langle u_{y}^{\prime 2} u_{z}^{\prime 2}\right\rangle /\left\langle u_{y}^{\prime 2}\right\rangle\left\langle u_{z}^{\prime 2}\right\rangle} \overline{\left\langle u_{x}^{\prime} u_{y}^{\prime 3}\right\rangle /\left\langle u_{x}^{\prime 2}\right\rangle^{1 / 2}\left\langle u_{y}^{\prime 2}\right\rangle^{3 / 2}}$} \\
\hline Isotropic & 2.82 & 2.80 & 2.79 & 0.94 & 0.97 & 0.08 \\
\hline Shear & 2.81 & 2.71 & 3.26 & 1.51 & 0.97 & 1.56 \\
\hline Convection & 2.99 & 2.58 & 2.93 & 1.37 & 1.01 & 1.26 \\
\hline DNS 3b [11] & 3.14 & 3.06 & - & - & - & - \\
\hline $\begin{array}{l}\text { Panchapakesan } \\
\text { and Lumley [23] }\end{array}$ & - & - & - & - & 0.97 & 1.23 \\
\hline
\end{tabular}

vorticity component magnitudes are given by $\left|\omega_{x}^{\prime}\right| / \omega_{\mathrm{rms}}=1.00,\left|\omega_{y}^{\prime}\right| / \omega_{\mathrm{rms}}=0.92$, and $\left|\omega_{z}^{\prime}\right| / \omega_{\mathrm{rms}}=$ 1.07. When shear convection is included, however, $\omega_{y}^{\prime}$ becomes the dominant vorticity component, and the average values are given by $\left|\omega_{x}^{\prime}\right| / \omega_{\text {rms }}=0.99,\left|\omega_{y}^{\prime}\right| / \omega_{\text {rms }}=1.07$, and $\left|\omega_{z}^{\prime}\right| / \omega_{\text {rms }}=0.94$.

Another significant impact of the shear convection on the flow is on the Reynolds shear stress. Figure 9(b) shows the Reynolds shear stress profile normalized by the turbulent kinetic energy for the shear and convection case. As mentioned earlier, for the shear case, the Reynolds shear stress has a value of $\bar{\beta} \simeq 0.41$, and the portion with shear convection shows a value of $\bar{\beta} \simeq 0.31$, which agrees better with results from the literature of shear-dominated turbulent flows such as mixing layers, planar jets, and round jets, as seen in Fig. 9(c).

\section{Higher order moments}

The average values of the third- and fourth-order moments of the velocity fluctuations are calculated for the three inflow/outflow simulations and tabulated along with round jet experimental results in Table II. These moments are related to the Gaussianity of the velocity field. More precisely, for a normal distribution, the skewness is zero and the flatness is 3. It can be seen that the skewness in $u_{x}^{\prime}$ and $u_{y}^{\prime}$ in the inflow/outflow simulations are also close to zero, and including convection does not significantly change the skewness results. It was shown by Dhandapani et al. [11] that simulations with only the linear forcing terms show no skewness, and to capture the skewness in the velocity, nonlinear forcing terms are needed in the simulations. The flatness of the velocity fluctuations are calculated as $\overline{\left\langle u_{i}^{\prime 4}\right\rangle /\left\langle u_{i}^{\prime 2}\right\rangle^{2}}$ and are close to the Gaussian value of 3 for all the simulations. Once again, there is no major difference in the flatness results between the three inflow/outflow simulations.

Some of the third- and fourth-order moments calculated are cross-correlations between different components of the velocity. The third-order cross-correlation moments are also near zero and confirm the previous observations by Dhandapani et al. [11]: nonlinear terms need to be included to get third moments to be nonzero. The fourth-order cross-correlation moments however, are nonzero. Using the quasi-Gaussian approximation, some of these moments can be predicted a priori, e.g., $\left\langle u_{x}^{\prime 2} u_{y}^{\prime 2}\right\rangle=\left\langle u_{x}^{\prime 2}\right\rangle\left\langle u_{y}^{\prime 2}\right\rangle+2\left\langle u_{x}^{\prime} u_{y}^{\prime}\right\rangle^{2},\left\langle u_{y}^{\prime 2} u_{z}^{\prime 2}\right\rangle=\left\langle u_{y}^{\prime 2}\right\rangle\left\langle u_{z}^{\prime 2}\right\rangle$, and $\left\langle u_{x}^{\prime} u_{y}^{\prime 3}\right\rangle=3\left\langle u_{x}^{\prime} u_{y}^{\prime}\right\rangle\left\langle u_{y}^{\prime 2}\right\rangle$. The normalized value of $\left\langle u_{x}^{\prime 2} u_{y}^{\prime 2}\right\rangle$ is close to 1 in the isotropic simulations as $u_{x}^{\prime}$ and $u_{y}^{\prime}$ are uncorrelated (i.e., $\left.\left\langle u_{x}^{\prime} u_{y}^{\prime}\right\rangle=0\right)$, and is higher than 1 in the shear and convection simulations as expected due to the 


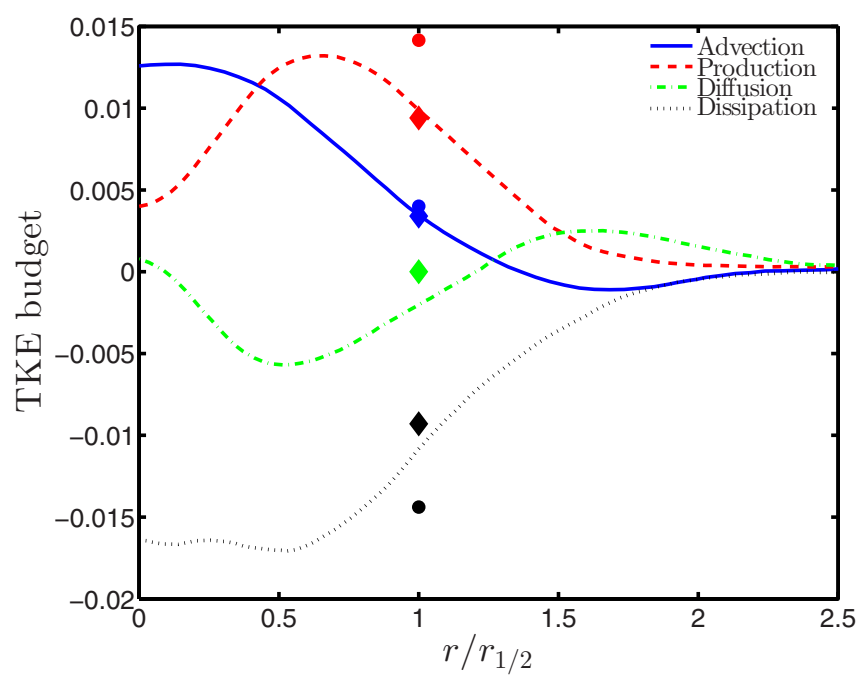

FIG. 10. Normalized turbulent kinetic energy budget. The lines correspond to experimental results from Panchapakesan and Lumley [23]. Symbols correspond to different simulations: with shear convection $=$ diamonds, without shear convection [DNS $3\left(192^{3}\right.$ grid $)$ from Dhandapani et al. [11] = circles.

cross-correlation. The normalized value of $\left\langle u_{y}^{\prime 2} u_{z}^{\prime 2}\right\rangle$ is near 1 for all the simulations and experiments, as $u_{y}^{\prime}$ and $u_{z}^{\prime}$ are always uncorrelated. Finally, the normalized value of $\left\langle u_{x}^{\prime} u_{y}^{\prime 3}\right\rangle$ is near zero for the isotropic simulations, and the convection simulation results agree very well with the experiments by Panchapakesan and Lumley [23]. Once again, the lower value of this fourth-order moment (when compared to the shear simulation without convection) is due to the smaller cross-correlation, $\left\langle u_{x}^{\prime} u_{y}^{\prime}\right\rangle$ (see Table I).

In summary, the addition of shear convection does not alter the higher order moments of the flow significantly. Third-order moments remain zero because of the linearity of the shear production and shear convection terms. Fourth-order moments can be predicted a priori from the Gaussianity of the velocity field and the $\left\langle u_{x}^{\prime} u_{y}^{\prime}\right\rangle$ cross-correlation term.

\section{Energy budget}

The turbulent kinetic energy budget values are calculated using the same method as in Dhandapani et al. and plotted in Fig. 10 with results from Dhandapani et al. [11] and the experiment results of Panchapakesan and Lumley [23]. All the values are normalized by $U_{o}^{3} / r_{1 / 2}$, where $U_{o}$ is calculated as $U_{o}=\sqrt{k / 0.052}$ [23] and $r_{1 / 2}$ is calculated as $r_{1 / 2}=0.586 U_{o} / B$ [25]. As expected, the shear convection term had zero contribution to the turbulent kinetic energy budget and is not included in the budget plot. It can be seen that the production, convection, and dissipation are accurately captured, compared to the simulation without convection.

\section{CONCLUSIONS}

The paper presented a series of direct numerical simulations (DNS) of increasing geometrical complexity to prove that statistically stationary, homogeneous shear turbulence (HST) simulations can be achieved. First, the impact of the aspect ratio of the computational domain on both isotropic and shear turbulence characteristics was studied. The integral length scale was slightly reduced in the elongated domains for the case of isotropic turbulence, while it remained the same for shear turbulence. The anisotropy in the turbulence remains unaffected by the aspect ratio of the computational domains. 
Introducing an inflow/outflow in one direction had very small effects on both isotropic turbulence and shear turbulence. The velocity and vorticity components remained isotropic for the isotropic turbulence forcing scheme and anisotropic for the shear turbulence, with very little change in the anisotropy values. The Reynolds shear stress was also not affected by the use of an inflow/outflow.

Shear convection was introduced in a region of the domain along with shear turbulence forcing. The inflow/outflow introduced in the nonhomogeneous direction was necessary to include shear convection without having to use shear periodic boundary conditions or other special boundary treatment. This resulted in statistically stationary homogeneous shear turbulence, which has remained elusive in most of the previous studies.

With shear convection included in the inflow/outflow simulations, the velocity anisotropy and Reynolds stress values agreed better with simulations and experiments of shear-dominated flows. The turbulent kinetic energy budget results also agreed better with those of free shear flows.

The present results underscore the necessity of using appropriate boundary conditions when simulating HST flows.

[1] R. S. Rogallo, Numerical experiments in homogeneous turbulence, NASA Technical Memorandum 81315 (1981).

[2] M. M. Rogers and P. Moin, The structure of the vorticity field in homogeneous turbulent flows, J. Fluid Mech. 176, 33 (1987).

[3] M. J. Lee, J. Kim, and P. Moin, Structure of turbulence at high shear rate, J. Fluid Mech. 216, 561 (1990).

[4] F. Baron, Three-dimensional macro-simulation of turbulent shear flows; Macro-simulation tridimensionnelle d'ecoulements turbulents cisailles, Ph. D. thesis, 1982.

[5] T. Gerz, U. Schumann, and S. E. Elghobashi, Direct numerical simulation of stratified homogeneous turbulent shear flows, J. Fluid Mech. 200, 563 (1989).

[6] K. A. Brucker, J. C. Isaza, T. Vaithianathan, and L. R. Collins, Efficient algorithm for simulating homogeneous turbulent shear flow without remeshing, J. Comput. Phys. 225, 20 (2007).

[7] J. C. Isaza and L. R. Collins, On the asymptotic behaviour of large-scale turbulence in homogeneous shear flow, J. Fluid Mech. 637, 213 (2009).

[8] M. H. Kasbaoui, R. G. Patel, D. L. Koch, and O. Desjardins, An algorithm for solving the NavierStokes equations with shear-periodic boundary conditions and its application to homogeneously sheared turbulence, J. Fluid Mech. 833, 687 (2017).

[9] A. Sekimoto, S. Dong, and J. Jiménez, Direct numerical simulation of statistically stationary and homogeneous shear turbulence and its relation to other shear flows, Phys. Fluids 28, 035101 (2016).

[10] P. L. Carroll and G. Blanquart, A proposed modification to Lundgren's physical space velocity forcing method for isotropic turbulence, Phys. Fluids 25, 105114 (2013).

[11] C. Dhandapani, K. J. Rah, and G. Blanquart, Effective forcing for direct numerical simulations of the shear layer of turbulent free shear flows, Phys. Rev. Fluids 4, 084606 (2019).

[12] K. J. Rah, C. Dhandapani, and G. Blanquart, Derivation of a realistic forcing term to reproduce the turbulent characteristics of round jets on the centerline, Phys. Rev. Fluids 3, 084606 (2018).

[13] T. S. Lundgren, Linearly forced isotropic turbulence, in Annual Research Briefs (Center for Turbulence Research, Stanford, 2003) pp. 461-473.

[14] O. Desjardins, G. Blanquart, G. Balarac, and H. Pitsch, High order conservative finite difference scheme for variable density low mach number turbulent flows, J. Comput. Phys. 227, 7125 (2008).

[15] S. B. Pope, Turbulent Flows (IOP Publishing, Cambridge, 2001).

[16] P. K. Yeung and S. B. Pope, Lagrangian statistics from direct numerical simulations of isotropic turbulence, J. Fluid Mech. 207, 531 (1989).

[17] B. Bobbitt, S. Lapointe, and G. Blanquart, Vorticity transformation in high Karlovitz number premixed flames, Phys. Fluids 28, 015101 (2016). 
[18] S. Lapointe and G. Blanquart, Fuel and chemistry effects in high Karlovitz premixed turbulent flames, Combust. Flame 167, 294 (2016).

[19] B. Savard and G. Blanquart, Broken reaction zone and differential diffusion effects in high Karlovitz $n-\mathrm{C}_{7} \mathrm{H}_{16}$ premixed turbulent flames, Combust. Flame 162, 2020 (2015).

[20] B. Savard, B. Bobbitt, and G. Blanquart, Structure of a high Karlovitz $n-\mathrm{C}_{7} \mathrm{H}_{16}$ premixed turbulent flame, Proc. Combust. Inst. 35, 1377 (2015).

[21] S. Lapointe, B. Savard, and G. Blanquart, Differential diffusion effects, distributed burning, and local extinctions in high Karlovitz premixed flames, Combust. Flame 162, 3341 (2015).

[22] C. Rosales and C. Meneveau, Linear forcing in numerical simulations of isotropic turbulence: Physical space implementations and convergence properties, Phys. Fluids 17, 095106 (2005).

[23] N. R. Panchapakesan and J. L. Lumley, Turbulence measurements in axisymmetric jets of air and helium. Part 1. Air jet, J. Fluid Mech. 296, 197 (1993).

[24] H. J. Hussein, S. P. Capp, and W. K. George, Velocity measurements in a high-Reynolds-number, momentum-conserving, axisymmetric, turbulent jet, J. Fluid Mech. 258, 31 (1994).

[25] H. Schlichting and K. Gersten, Boundary-Layer Theory (Springer, Berlin, 2016). 